\title{
Detection of Cancer Using Vector Quantization for Segmentation
}

\author{
Dr. H. B. Kekre \\ Computer \\ Engineering \\ MPSTME, NMIMS \\ University, \\ Vileparle(w) \\ Mumbai 400-056, \\ India
}

\author{
Dr.Tanuja Sarode \\ Assistant Professor \\ TSEC, Bandra(W), \\ Mumbai - 50, India \\ Ms.Saylee Gharge \\ Assistant Professor, \\ V.E.S.I.T, \\ Mumbai-400071, \\ India
}

\author{
Ms. Kavita Raut \\ Lecturer \\ Thadomal Shahani \\ Engineering College, \\ Bandra $(\mathrm{W})$, \\ Mumbai - 50, India
}

\begin{abstract}
:
Breast cancer is one of the major causes of death among women. An improvement of early diagnostic techniques is critical for women's quality of life. Mammography is the main test used for screening and early diagnosis. Contrast-enhanced magnetic resonance of the breast is the most attractive alternative to standard mammography. This paper presents a vector quantization segmentation method to detect cancerous mass from mammogram images. In order to increase radiologist's diagnostic performance, several computer-aided diagnosis (CAD) schemes have been developed to improve the detection of primary signatures of this disease: masses and microcalcifications.
\end{abstract}

\section{Keywords:}

Mammography, Segmentation, Vector Quantization, Clustering.

\section{INTRODUCTION:}

Breast cancer can be most effectively treated when it is detected at its early stage. Ultrasonography is widely used for the detection and evaluation of many diseases. In the case of breast cancer, it is a very useful complementary imaging technique to mammography. The most effective method for early detection and screening of breast cancers is X-ray mammography. However, reading mammography is a demanding job for radiologists, and cannot provide consistent results from time to time. The judgments depend on training, experience, and subjective criteria. In addition, mammography produces a high false positive rate, and only about 525 of 1800 lesions [1].

Segmentation will help in the computer-aided evaluation of the tumors and the distinction of benign and malignant nodules. Since screening mammography is currently the main test for early detection of breast cancer, a huge number of mammograms need to be examined by a limited number of radiologists. In order to improve the diagnostic efficiency, computer-aided diagnosis has been introduced into the screening process [2].

Mammography is the main test used for screening and early diagnosis. Early detection performed on X-ray mammography is the key to improve breast cancer prognosis. In order to increase radiologist's diagnostic performance, several computer-aided diagnosis (CAD) schemes have been developed to improve the detection of primary signatures of this disease: masses and microcalcifications [3].
A number of image processing methods have been proposed to perform this task. S. M. Lai et al [4] and W. Qian et al [5] have proposed using modified and weighted median filtering, respectively, to enhance the digitized image prior to object identification. D. Brzakovic et all [6] used thresholding and fuzzy pyramid linking for mass localization and classification. Other investigators have proposed using the asymmetry between the right and left breast images to determine possible mass locations. Yin et al. uses both linear and nonlinear bilateral subtraction [7] while the method by Lau et al. [8] relies on "structural asymmetry" between the two breast images. Recently Kegelmeyer [9] has reported promising results for detecting speculated lesions based on local edge characteristics and Laws texture features [10, 11].The above methods produced a true positive detection rate of approximately $90 \%$. The work we have done till now is to propose a segmentation process which identifies on a mammogram the opaque areas, suspect or not, present in the image using vector quantization [12-21].

Tumors or calcifications are embedded in an inhomogeneous background. In mammograms, background objects may even appear brighter. Therefore, global threshold methods suffer considerable drawback. The work we have done is to propose a segmentation process which identifies on a mammogram the opaque areas, suspect or not, present in the image using vector quantization which consumes moderate time but provide good accuracy with less complexity. Watershed algorithm has a drawback of over-segmenting the image making it obscure for identification of tumor. Segmentation using gray level cooccurrence matrix required huge time for tumor demarcation with less accuracy. Vector quantization segmentation algorithm attempts to overcome such drawbacks.

\subsection{Vector Quantization:}

Vector quantization is a classical quantization technique from signal processing which allows the modeling of probability density functions by the distribution of prototype vectors. It was originally used for data compression. It works by dividing a large set of points (vectors) into groups having approximately the same number of points closest to them. Each group is represented by its centroid point as in K-means some and other clustering algorithms.

The density matching property of vector quantization is powerful, especially for identifying the density of large and highdimensioned data. Since data points are represented by the index 
of their closest centroid, commonly occurring data have low error, and rare data high error. This is why VQ is suitable for lossy data compression. It can also be used for lossy data correction and density estimation [6].

Vector Quantization (VQ) [22-30] is an efficient technique for data compression and has been successfully used in various applications such as index compression [31, 32]. VQ has been very popular in a variety of research fields such as speech recognition and face detection [33, 34]. VQ is also used in real time applications such as real time video-based event detection [35] and anomaly intrusion detection systems [36], image segmentation [37-40], speech data compression [41], content based image retrieval CBIR [42] and face recognition [43].

The rest of the paper is organized as follows. Section 2 describes Gray Level Co-occurrence Matrix (GLCM), Watershed algorithm and Kekre's Median Codebook Generation (KMCG) algorithm used for image segmentation of mammographic images. Followed by the experimental results for mammographic images for comparison in section 3 and section 4 concludes the work.

\section{ALGORITHMS FOR SEGMENTATION}

In this section we explain segmentation by Gray level cooccurrence matrix [44], basic watershed algorithm [45-49] and Kekre's Median Codebook Generation (KMCG) algorithm which are used for comparative performance of tumor detection.

\subsection{Gray Level Co-occurrence Matrix}

Haralick [50] suggested the use of gray level co-occurrence matrices (GLCM) for definition of texture features. The values of the co-occurrence matrix elements present relative frequencies with which two neighboring pixels separated by distance $d$ appear on the image, where one of them has gray level $i$ and other $j$. Such matrix is symmetric and also a function of the angular relationship between two neighboring pixels. The co-occurrences matrix can be calculated on the whole image, but by calculating it in a small window which scanning the image, the co-occurrence matrix can be associated with each pixel. By using gray level co-occurrence matrix we can extract different features like probability, entropy, energy, variance, inverse moment difference etc. Using cooccurrence matrix the major textural features are defined as:

Maximum Probability: $\max \left(\mathrm{P}_{\mathrm{ij}}\right)$

Variance:

$$
\left(\sum\left(\mathrm{i}-\mu_{\mathrm{i}}\right)^{2} \sum \mathrm{P}_{\mathrm{ij}}\right)\left(\sum\left(\mathrm{j}-\mu_{\mathrm{j}}\right)^{2} \sum \mathrm{P}_{\mathrm{ij}}\right)
$$

Correlation:

$$
\sum_{i} \sum_{j}\left(i-\mu_{x}\right)\left(j-\mu_{y}\right) P_{i j} / \sigma_{x} \sigma_{y}
$$

where $\mu_{\mathrm{x}}$ and $\mu_{\mathrm{y}}$ are means and $\sigma_{\mathrm{x}}, \sigma_{\mathrm{y}}$ are standard deviation

$$
\text { Entropy: } \sum_{\mathrm{i}} \sum_{\mathrm{j}} \mathrm{P}_{\mathrm{ij}} \log \left(\mathrm{P}_{\mathrm{ij}}\right)
$$

Amongst all these features entropy has given us the best results. Hence in this paper we extracted entropy using gray level co- occurrence matrix and the results are displayed in Fig.5 (b) along with that of watershed and KMCG algorithms for comparison.

\subsection{Watershed Algorithm}

Watershed segmentation [48] classifies pixels into regions using gradient descent on image features and analysis of weak points along region boundaries. The image feature space is treated, using a suitable mapping, as a topological surface where higher values indicate the presence of boundaries in the original image data. It uses analogy with water gradually filling low lying landscape basins. The size of the basins grows with increasing amount of water until they spill into one another. Small basins (regions) gradually merge together into larger basins. Regions are formed by using local geometric structure to associate the image domain features with local extremes measurement. Watershed techniques produce a hierarchy of segmentations, thus the resulting segmentation has to be selected using either some a priory knowledge or manually. These methods are well suited for different measurements fusion and they are less sensitive to user defined thresholds. We implemented watershed algorithm for mammographic images as mentioned in [2].Results for mammographic images are displayed in Fig 5(c).

\subsection{Proposed Algorithm}

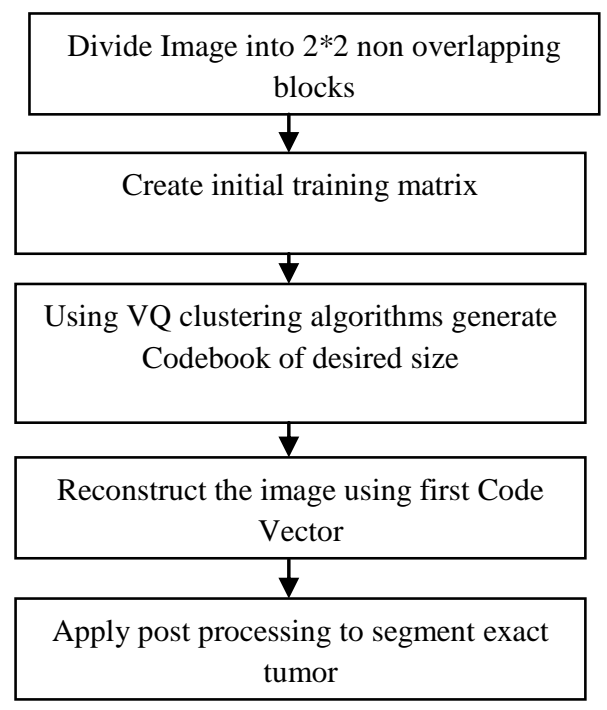

Fig. 1 Block Diagram of Proposed Algorithm

\section{Steps for the algorithm}

Step1: Divide the image into non overlapping blocks.

Step2: Convert each block into training vector. Entire Training matrix is considered as one cluster $(\mathrm{X})$

Step3: Codebook of desired size is generated using KMCG.

Step 4: Reconstruct image using first codevector

Step 5: Post processing is applied on reconstructed image to segment exact tumor

\subsubsection{Kekre's Median Fast Codebook Generation (KMCG) Algorithm [26]:-}

In this algorithm image is divided in to blocks and blocks are converted to the vectors of size k. The Fig. 2 below represents matrix $\mathrm{T}$ of size $\mathrm{Mx} \mathrm{k}$ consisting of $\mathrm{M}$ number of image training vectors of dimension $\mathrm{k}$. 
Each row of the matrix is the image training vector of dimension $\mathrm{k}$.

$$
\mathrm{T}=\left[\begin{array}{cccc}
\mathrm{x}_{1,1} & \mathrm{x}_{1,2} & \ldots . & \mathrm{x}_{1, \mathrm{k}} \\
\mathrm{x}_{2,1} & \mathrm{x}_{2,2} & \ldots . & \mathrm{x}_{2, \mathrm{k}} \\
\cdot & \cdot & \cdot & \cdot \\
\cdot & \cdot & \cdot & \cdot \\
\mathrm{x}_{M, 1} & \mathrm{x}_{M, 2} & \ldots . & \mathrm{x}_{M, k}
\end{array}\right]
$$

Fig.2 Matrix of image training vector

The training vectors are sorted with respect to the first member of all the vectors i.e with respect to the first column of the matrix $\mathrm{T}$ and the entire matrix is considered as one single cluster. The median of the matrix $\mathrm{T}$ is chosen (codevector) and is put into the codebook, and the size of the codebook is set to one. The matrix is then divided into two equal parts and the each of the part is then again sorted with respect to the second member of all the training vectors i.e. with respect to the second column of the matrix $\mathrm{T}$ and we obtain two clusters both consisting of equal number of training vectors. The median of both the parts is the picked up and written to the codebook, now the size of the codebook is increased to two consisting of two codevectors and again each part is further divided to half. Each of the above four parts obtained are sorted with respect to the third column of the matrix $\mathrm{T}$ and four clusters are obtained and accordingly four codevectors are obtained. The above process is repeated till we obtain the codebook of desired size. Here quick sort algorithm is used.

\subsubsection{Post processing}

In post processing step the image obtained after VQ segmentation is taken as input image. The VQ segmented image is labeled and area of each region is calculated. The region with larger area is extracted. The boundary of extracted region is superimposed on original image. Results for proposed method on mammographic images are displayed in Fig 3(a-e) and Fig.4 (a-e).

\section{RESULTS:}

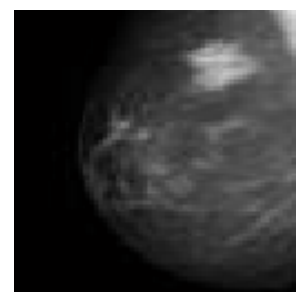

(a)

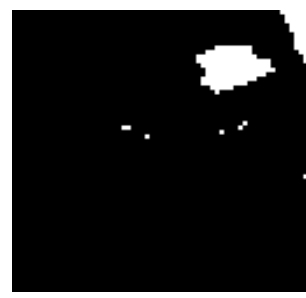

(b)

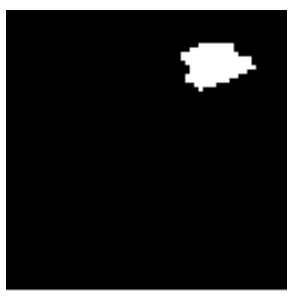

(c)

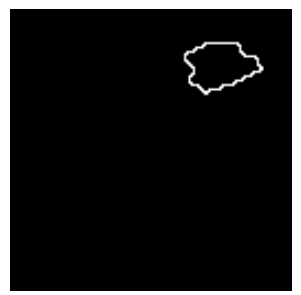

(d)

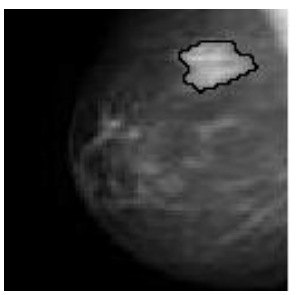

(e)

Fig.3: (a) Original Breast tumor Mammogram Image,(b) Image after VQ segmentation, (c) ROI Extracted Image,(d)Boundary Extracted Image, (e) Superimposed Image

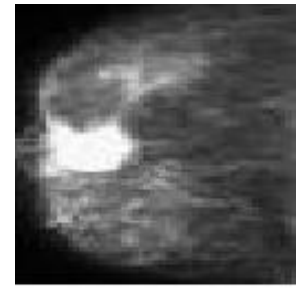

(a)

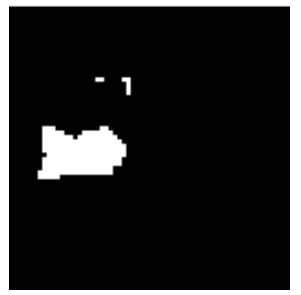

(b

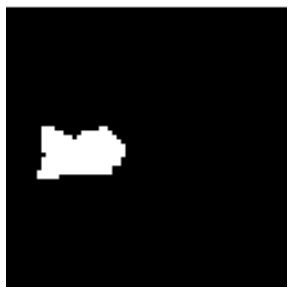

(c)

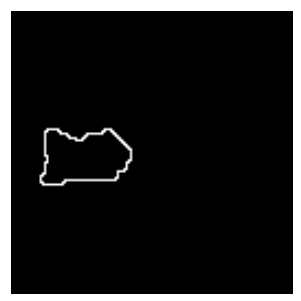

(d)

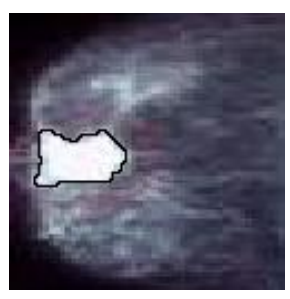

(e)

Fig.4: (a) Original Breast tumor Mammogram Image,(b) Image after VQ segmentation, (c) ROI Extracted Image,(d)Boundary Extracted Image, (e) Superimposed Image 


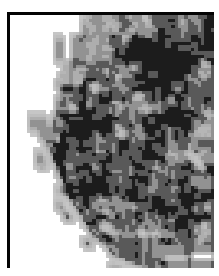

(a)

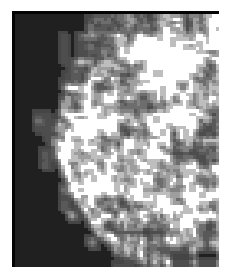

(b)

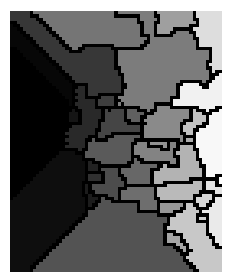

(c)
Fig.5: (a) Probability image using GLCM, (b) Entropy image using GLCM, (c) Segmented image using Watershed algorithm

\section{CONCLUSION:}

Mammograms contain low signal to noise ratio (low contrast) and a complicated structured background. Breast tissue contrast and density vary with age, thus mammography produces varying image qualities. In addition, mammographic images are not bimodal. As a result, any segmentation method which utilizes an a-priori or single threshold value method is highly likely to generate serious segmentation errors. Moreover, tumors or calcifications are embedded in an inhomogeneous background. In mammograms, background objects may even appear brighter. Therefore, global threshold methods suffer considerable drawback.

Vector quantization segmentation algorithm attempts to overcome such drawbacks. Vector quantization is based on clustering algorithm. It is observed form the results that the KMCG algorithm takes least time to generate codebook, since it does not require Euclidean distance computation. The programs are tested on thirty five mammogram images. Identification rate for proposed method is $68.5 \%$.

\section{REFERENCES:}

[1]. Parveen, N.R.S, "Segmenting Tumors in Ultrasound Images," Proceeding of the 2008 International Conference on Computing, Communication and Networking, pp.: 1-5, 2008.

[2]. Basim Alhadidi, Mohammad H. Zu bi and Hussam N. Suleiman, "Mammogram Breast Cancer Image Detection Using Image Processing Functions," Information Technology Journal, Volume: 6, Issue: 2, pp.: 217-221, 2007.

[3]. S. Saheb Basha, Dr. K. Satya Prasad, "Automatic Detection Of Breast cancer mass in mammograms using morphological Operators and Fuzzy C-Means," Journal of Theoretical and Applied Information Technology, pp.: 704-709, 2005.

[4]. J. Dengler, S. Behrens, and J. F. Desaga, "Segmentation of microcalcifications in mammograms," IEEE Trans. Med. Imag., vol. 12, no. 4, pp. 634-642, Dec. 1993.

[5]. D. Zhao, "Rule-based morphological feature extraction of microcalcifications in mammograms," SPIE Med. Imag., vol. 1095, pp. 702-715,1993.

[6]. S. C. Lo, H. P. Chan, J. S. Lin, H. Li, M. T. Freedman, and S. K. Mun, "Artificial convolution neural network for medical image pattern recognition," Neural Networks, vol.8, no. 7/8, pp. 1201-1214, 1995.

[7]. W. Zhang, K. Doi, M. L. Giger, Y. Wu, R. M. Nishikawa, and R. A. Schmidt, "Computerized detection of clustered microcalcifications in digital mammograms using a shiftinvariant artificial neural network," Med. Phys., vol. 21, no. 4, pp. 517-524, 1994.

[8]. Y. Wu, K. Doi, M. L. Giger, and M. Nishikawa, "Computerized detection of clustered microcalcifications in digital mammograms: Application of artificial neural networks," Med. Phys., vol. 19, pp. 555-560, 1992.

[9]. N. Karssemeijer, "A stochastic model for automated detection of calcifications in digital mammograms," in Proc. 12th Int. Conf. IPMI, Wye,UK, 1991, pp. 227238.

[10].N. Karssemeijer, "Recognition of clustered microcalcifications using a random field model," SPIE Med. Imag., vol. 1905, pp. 776-786, 1993.

[11].F. Lefebvre, H. Benali, R. Gilles, E. Kahn, and R. D. Paola, "A fractal approach to the segmentation of microcalcification in digital mammograms," Med. Phys.,vol. 22, no. 4, pp. 381-390, 1995.

[12].D. Brzakovic, P. Brzakovic, and M. Neskovic, "An approach to automated screening of mammograms," SPIE Biomed. Image Processing, Biomed. Visual. vol. 1905, pp. 690-701, 1993.

[13].H. Yoshida, K. Doi, and R. M. Nishikawa, "Automated detection of clustered microcalcifications in digital mammograms using wavelet transform techniques," SPIE Image Processing, vol. 2167, pp. 868-886, 1994.

[14].A.F.Laine, S. Schuler, J. Fan, and W. Huda,"Mammographic feature enhancement by multiscale analysis," IEEE Trans. Med. Imag., vol. 13, no. 4, pp. 725-740, Dec. 1994.

[15].I. N. Bankman, W. A. Christens-Brry, D. W. Kim, I. N. Weinberg, O. B. Gatewood, and W. R. Brody, "Automated recognition of microcalcification clusters in mammograms,"SPIE Biomed. Image Processing, Biomed. Visual., vol.1905, pp. 731-738, 1993.

[16].Tou, J., and Gonzalez, Pattern Recognition Principles Addison-Wesley Publishing Company 1974.

[17].Chen, J., Kundu A. "Unsupervised texture segmentation using multichannel decomposition and hidden markov models", IEEE Transactions on Image Processing 4(5):603-619, 1994.

[18].Pemmaraju, S., Mitra S., Shieh Y., Roberson G. "Segmentation of radiographic cervical images with neurofuzzy classification of multiresolution wavelets", Proceedings of SPIE Medical Imaging -1995

[19].Dr. H. B. Kekre, Saylee Gharge ,"Image Segmentation of MRI using Texture Features," International Conference on Managing Next Generation Software Applications, School of Science and Humanities, Karunya University, Coimbatore, Tamilnadu, 05-06 December, 2008.

[20].Dr. H. B. Kekre , Saylee Gharge , "Statistical Parameters like Probability and Entropy applied to SAR image segmentation," International Journal of Engineering Research \& Industry Applications (IJERIA), Vol.2,No.IV,pp.341-353.

[21].R. M. Gray, "Vector quantization", IEEE ASSP Mag., pp.: 4-29, Apr. 1984 
[22].Y. Linde, A. Buzo, and R. M. Gray, "An algorithm for vector quantizer design," IEEE Trans.Commun., vol. COM- 28, no. 1, pp.: 84-95, 1980

[23].H.B.Kekre, Tanuja K. Sarode, "New Fast Improved Clustering Algorithm for Codebook Generation for Vector Quantization", International Conference on Engineering Technologies and Applications in Engineering, Technology and Sciences, Computer Science Department, Saurashtra University, Rajkot, Gujarat. (India), Amoghsiddhi Education Society, Sangli, Maharashtra (India), 13th - 14th January 2008.

[24].H. B. Kekre, Tanuja K. Sarode, "New Fast Improved Codebook Generation Algorithm for Color Images using Vector Quantization," International Journal of Engineering and Technology, vol.1, No.1, pp.: 67-77, September 2008.

[25].H. B. Kekre, Tanuja K. Sarode, "Fast Codebook Generation Algorithm for Color Images using Vector Quantization," International Journal of Computer Science and Information Technology, Vol. 1, No. 1, pp.: 7-12, Jan 2009.

[26].H. B. Kekre, Tanuja K. Sarode, "An Efficient Fast Algorithm to Generate Codebook for Vector Quantization," First International Conference on Emerging Trends in Engineering and Technology, ICETET-2008, held at Raisoni College of Engineering, Nagpur, India, pp.: 62- 67, 16-18 July 2008. Avaliable at IEEE Xplore.

[27].H. B. Kekre, Tanuja K. Sarode, "Fast Codebook Generation Algorithm for Color Images using Vector Quantization," International Journal of Computer Science and Information Technology, Vol. 1, No. 1, pp.: 7-12, Jan 2009.

[28].H. B. Kekre, Tanuja K. Sarode, "Fast Codevector Search Algorithm for 3-D Vector Quantized Codebook", WASET International Journal of cal Computer Information Science and Engineering (IJCISE), Volume 2, No. 4, pp.: 235-239, Fall 2008. Available: http://www.waset.org/ijcise.

[29].H. B. Kekre, Tanuja K. Sarode, "Fast Codebook Search Algorithm for Vector Quantization using Sorting Technique", ACM International Conference on Advances in Computing, Communication and Control (ICAC3-2009), pp: 317-325, 23-24 Jan 2009, Fr. Conceicao Rodrigous College of Engg., Mumbai. Available on ACM portal.

[30].Jim Z.C. Lai, Yi-Ching Liaw, and Julie Liu, "A fast VQ codebook generation algorithm using codeword displacement", Pattern Recogn. vol. 41, no. 1, pp.: 315319,2008 .

[31].C.H. Hsieh, J.C. Tsai, Lossless compression of VQ index with search order coding, IEEE Trans. Image Process. vol. 5, No. 11, pp.: 1579-1582, 1996.

[32]. Chin-Chen Chang, Wen-Chuan Wu, "Fast PlanarOriented Ripple Search Algorithm for Hyperspace VQ Codebook", IEEE Transaction on image processing, vol 16, no. 6, pp.: 1538-1547, June 2007.

[33].C. Garcia and G. Tziritas, "Face detection using quantized skin color regions merging and wavelet packet analysis,"= IEEE Trans. Multimedia, vol. 1, no. 3, pp.: 264-277, Sep. 1999.
[34].H. Y. M. Liao, D. Y. Chen, C. W. Su, and H. R. Tyan, "Real-time event detection and its applications to surveillance systems," in Proc. IEEE Int. Symp. Circuits and Systems, Kos, Greece, pp.: 509-512, May 2006.

[35].J. Zheng and M. Hu, "An anomaly intrusion detection system based on vector quantization," IEICE Trans. Inf. Syst., vol. E89-D, no. 1, pp.: 201-210, Jan. 2006.

[36].H. B. Kekre, Tanuja K. Sarode, Bhakti Raul, "Color Image Segmentation using Kekre's Fast Codebook Generation Algorithm Based on Energy Ordering Concept", ACM International Conference on Advances in Computing,Communication and Control (ICAC32009), pp.: 357-362, 23-24 Jan 2009, Fr. Conceicao Rodrigous College of Engg., Mumbai. Available on ACM portal.

[37].H. B. Kekre, Tanuja K. Sarode, Bhakti Raul, "Color Image Segmentation using Kekre's Algorithm for Vector Quantization", International Journal of Computer Science(IJCS), Vol. 3, No. 4, pp.: 287 292,Fall2008. Available:http://www.waset.org/ijcs.

[38].H. B. Kekre, Tanuja K. Sarode, Bhakti Raul, "Color Image Segmentation using Vector Quantization Techniques Based on Energy Ordering Concept" International Journal of Computing Science and Communication Technologies (IJCSCT) Volume 1, Issue 2, pp: 164-171, January 2009.

[39].H. B. Kekre, Tanuja K. Sarode, Bhakti Raul, "Color Image Segmentation Using Vector Quantization Techniques",Advances in Engineering Science Sect. C (3), pp.: 35-42,July-September 2008.

[40].H. B. Kekre, Tanuja K. Sarode, "Speech Data Compression using Vector Quantization", WASET International Journal of Computer and Information Science and Engineering (IJCISE), vol. 2, No. 4, pp.: 251-254, Fall 2008. Available: http://www.waset.org/ijcise.

[41].H. B. Kekre, Ms. Tanuja K. Sarode, Sudeep D. Thepade,"Image Retrieval using Color-Texture Features from DCT on VQ Codevectors obtained by Kekre's Fast Codebook Generation", ICGST-International Journal on Graphics, Vision and Image Processing (GVIP), Volume 9, Issue 5, pp.: 1-8, September 2009. Available online.http://www.icgst.com/gvip/Volume9/Issue5/P115 0921752.

[42].H. B. Kekre, Kamal Shah, Tanuja K. Sarode, Sudeep D. Thepade,'Performance Comparison of Vector Quantization Technique - KFCG with LBG, Existing Transforms and PCA for Face Recognition", International Journal of Information Retrieval (IJIR), Vol. 02, Issue 1, pp.: 64-71,2009.

[43].Dr. H. B. Kekre , Saylee Gharge , "SAR Image Segmentation using co-occurrence matrix and slope magnitude," ACM International Conference on Advances in Computing, Communication and Control (ICAC3-2009), pp.: 357-362, 23-24 Jan 2009, Fr. Conceicao Rodrigous College of Engg., Mumbai. Available on ACM portal.

[44].L. Vincent, P. Soille, Watersheds in digital spaces: An efficient algorithm based on immersion Simulations, IEEE Trans. PAMI., 13 (6) (1991) 583-593.

[45].F. Meyer, Topographic distance and watershed lines,Signal Processing, 38 (1) (1994) 113-125. 
[46].A. Bieniek, A. Moga, An efficient watershed algorithm based on connected components, Pattern Recognition, 33 (6) (2000) 907-916.

[47].M. Frucci, Oversegmentation reduction by flooding regions and digging watershed lines, International Journal of Pattern Recognition and Artificial Intelligence,

[48].L. E. Band, Topographic partition of watersheds with digital elevation models, Water Resources Res., 22 (1) (1986) 15-24.

[49].Leila Shafarenko and Maria Petrou, "Automatic Watershed Segmentation of Randomly Textured Color Images", IEEE Transactions on Image Processing, Vol.6, No.11, pp.1530- 1544, 1997.

[50].Robert M. Haralick, Statistical and Structural Approaches to Texture, IEEE Proceedings Of vol. 67, no. 5, May 1979.

\section{AUTHOR BIOGRAPHIES}

Dr. H. B. Kekre has received B.E. (Hons.) in Telecomm. Engg. from Jabalpur University in 1958, M.Tech(Industrial Electronics) from IIT Bombay in 1960, M.S.Engg. (Electrical Engg.) from University of Ottawa in 1965 and Ph.D. (System Identification) from IIT Bombay in 1970. He has worked Over 35 years as Faculty of Electrical Engineering and then HOD Computer Science and Engg. at IIT Bombay. For last 13 years worked as a Professor in Department of Computer Engg. at Thadomal Shahani Engineering College, Mumbai. He is currently senior Professor working with Mukesh Patel School of Technology Management and Engineering, SVKM's NMIMS University, Vile Parle(w), Mumbai, INDIA. His areas of interest are Digital Signal processing, Image Processing and computer networks. He has more than 250 papers in National / International Conferences / Journals to his credit. Recently six students working under his guidance have received best paper awards. Currently he is guiding ten Ph.D. students.
Dr.Tanuja K. Sarode has Received Ph.D. from Mukesh Patel School of Technology, Management and Engg. SVKM's NMIMS University, Vile-Parle (W), Mumbai, INDIA. She has more than 10 years of experience in teaching. She is currently working as Assistant Professor in Dept. of Computer Engineering at Thadomal Shahani Engineering College, Mumbai. She is life member of IETE, member of International Association of Engineers (IAENG) and International Association of Computer Science and Information Technology (IACSIT), Singapore. Her areas of interest are Image Processing, Signal Processing and Computer Graphics. She has 50 papers in National /International Conferences/journal to her credit.

Ms. Saylee M. Gharge has received M.E. (Electronics and telecomm.) degree from Mumbai University in 2007, currently Pursuing Ph.D. from Mukesh Patel School of Technology, Management and Engineering, NMIMS University, Vile-Parle (W), Mumbai. She has more than 9 years of experience in teaching. She is currently working as a lecturer in department of electronics and telecommunication in Vivekanand Institute of Technology, Mumbai. Her areas of interest are Image Processing, Signal Processing. She has 19 papers in National /International Conferences/journal to her credit.

Ms. Kavita C.Raut has received B.E. (Computer Engg.) degree from Mumbai University in 2005, currently Pursuing M.E. from Thadomal Shahani Engineering College, Bandra (W), Mumbai. She has more than 4 years of experience in teaching. She is currently working as a lecturer in department of Computer Engineering at Thadomal Shahani Engineering College, Mumbai. 